\title{
Publisher's Note: Quantification of Einstein-Podolsky-Rosen steering for two-qubit states [Phys. Rev. A 93, 020103(R) (2016)]
}

\author{
A. C. S. Costa and R. M. Angelo 1
}

(2) (Received 29 August 2019; published 5 September 2019)

DOI: 10.1103/PhysRevA.100.039901

This paper was published online on 19 February 2016 with a misspelling in the title and throughout the article. The title should read as "Quantification of Einstein-Podolsky-Rosen steering for two-qubit states." All instances of "Podolski" throughout the article should read as "Podolsky." The paper has been corrected as of 8 August 2019. The text is incorrect in the printed version of the journal. 